\title{
SERUM AMINOTRANSFERASES LEVEL IN PATIENTS WITH TYPE 2 DIABETES MELLITUS ATTENDING A TERTIARY CARE CENTER, KATHMANDU
}

\author{
Mainali A, Uprety N, Adhikari P, Pathak UN
}

Department of Internal Medicine, Nepal Medical College and Teaching Hospital,

Attarkhel, Gokarneshwor-8, Kathmandu, Nepal

\begin{abstract}
Nonalcoholic fatty liver disease is increasing with the rise in obesity and metabolic risk factors like diabetes mellitus. Type 2 diabetics are highly prone to deranged liver function test. Increase in serum aminotransferases level is an indicator of hepatocellular injury. The aim of this study is to correlate serum aminotransferases level with anthropometric measurements, diabetic retinopathy and types of treatment received in diabetics. A total of 236 patients (142 females) of age more than 40 years with type 2 diabetes attending Nepal Medical College and Teaching Hospital between February 2016 and January 2017 were included in this cross-sectional observational study. Blood samples were sent for fasting blood sugar, HbA1c and serum aminotransferases tests. Body mass index and waist hip ratio were measured as per standard protocol. Fundoscopy was done in all patients. Pearson and independent t-test were applied to see the correlation between the variables. The mean HbA1c was $8.01 \pm 2.08$. In the sub group analysis, serum aminotransferases level was significantly high in patients with poor glycemic control, higher body mass index and waist hip ratio ( $p$ value $<0.001$ ). Patients with diabetic retinopathy also had higher serum aminotransferases level ( $p$ value $<0.001$ ). Pearson correlation between HbA1c with serum alanine aminotransferases level was significant $(r=0.76, n=5$, $\mathrm{p}$ value $<0.001)$.
\end{abstract}

\section{KEYWORDS}

Body Mass Index, nonalcoholic

fatty liver disease, serum aminotransferases, waist hip ratio

\section{CORRESPONDING AUTHOR}

Dr. Arjun Mainali,

Department of Internal Medicine,

Nepal Medical College and Teaching Hospital,

Attarkhel, Gokarneshwor-8, Kathmandu, Nepal

Email: arjunmainali56@gmail.com 


\section{INTRODUCTION}

Diabetes mellitus is one of the major noncommunicable diseases and the prevalence is rising globally. ${ }^{1}$ The prevalence of diabetes worldwide was estimated to be $2.8 \%$ in 2000 and $4.4 \%$ in 2030 . It is projected to increase from 171 million in 2000 to 366 million in 2030. ${ }^{2}$ In Nepal, diabetes is an endemic disease, and is bringing new challenges in connection with rapid urbanization and modernization. ${ }^{3}$ The study done by Nepal Diabetes Association in urban areas has revealed a prevalence of diabetes of $15 \%$ among population 20 years and above and $19 \%$ among population above 40 years. ${ }^{4}$ Nearly 70 to $80 \%$ of the diabetic subjects have been reported to have hepatic fat accumulation, referred to as Nonalcoholic Fatty Liver (NAFL). ${ }^{5}$ NAFL leads to nonalcoholic steatohepatitis (NASH), a progressive fibrotic disease, which can result in cirrhosis or liver related death. ${ }^{6}$ The prevalence of NAFLD and NASH in United States is found to be 10 to 46 percent and 3 to 5 percent respectively. ${ }^{7,8}$ The prevalence of NAFLD in Asia-Pacific regions is estimated to range from 5 to 30 percent. ${ }^{9}$ There is renewed interest in NAFLD recently because of the increased prevalence in diabetes and as it has been shown to be associated with insulin resistance and hyperinsulinemia. ${ }^{10}$ Insulin resistance has been observed in patients with nonalcoholic steatohepatitis (NASH) who are not obese and those who have normal glucose tolerance. ${ }^{11}$ Significantly increased FFA levels have been observed in patients with NAFLD and type 2 diabetes mellitus, compared with type 2 diabetics without NAFLD. ${ }^{12}$ The most widely accepted theory implicates insulin resistance as the key mechanism leading to hepatic steatosis, and perhaps also to steatohepatitis. The insulin resistant state is also marked by an increase in proinflammatory cytokines such as tumor necrosis factor-a (TNF-a), which may also contribute to hepatocellular injury. ${ }^{13}$ Increased activities of serum aminotransferases are indicators of hepatocellular injury and increased activity of these markers are associated with insulin resistance, ${ }^{14}$ metabolic syndrome, and type 2 diabetes mellitus. Currently, screening for NAFLD is not recommended for patients at increased risk

\section{MATERIALS AND METHODS}

This is a cross-sectional observational study conducted in Nepal Medical College Teaching Hospital, Jorpati, Kathmandu. All inpatients and outpatients with diabetes mellitus of age more than 40 years irrespective of glucose control and treatment received (OHA/Insulin) were included in the study. The study was conducted for a period of 1 year (February 2016 to January 2017); a total of 236 patients were enrolled in the study after getting their consent. Those cases with gestational diabetes, type 1 diabetes mellitus, steroids induced diabetes, diabetic patients with history of alcohol intake, hepatotoxic drugs like amiodarone, antitubercular drugs, history of liver diseases or clinical evidence of acute hepatitis, those who were found to have evidence of hepatitis B and C virus infection (HBsAg positive and HCV antibody positive) and patients with congestive heart failure were excluded from this study. Patients in the study underwent a full clinical evaluation. Clinical history and physical examination findings were recorded with particular attention to anthropometric measurement like height, weight, waist and hip circumference. Weight and height were measured using standard procedures and Body Mass Index (BMI) was then calculated from the formula of weight $(\mathrm{kg}) / \mathrm{height}$ $\left(\mathrm{m}^{2}\right)$. Waist was measured at the midpoint between lower costal margin and superior iliac crest as per WHO guideline. Similarly, hip circumference was measured from the widest part of the buttock. Waist hip ratio was then calculated taking the cut off value for the Asian population. All patients underwent blood tests for serum aminotransferases, fasting blood sugar, post prandial blood sugar and HbA1c. Five millilitres of venous blood sample was taken during fasting. Serum aminotransferases and HbA1c levels measured in fasting blood sample. Fasting blood sugar was determined by using glucose oxidaseperoxidase test. Serum aminotransferases level was determined by Vitros 250 chemical analyzer by filer photometric method. HbA1c was determined by standard laboratory method as certified by National Glycohemoglobin Standarization program (NGSP). All data were entered into a proforma and results were analyzed by SPSS 16 using Pearson test and independent t-test.

\section{RESULTS}

Of the total 236 patients, 142 (60.2\%) were females and $94(39.8 \%)$ were males. Among all age groups maximum patients were of $41-50$ years $(n=93$, $39.4 \%)$

\begin{tabular}{|lccc|}
\multicolumn{4}{|c|}{ Table 1: Baseline characteristics } \\
Characteristics & Min & Max & Mean \pm SD \\
Age & 41 & 89 & 52 \\
BMI & 18.5 & 32.5 & 23.5 \\
Waist Hip Ratio & 0.81 & 0.95 & 0.87 \\
(Male) & & & \\
Waist Hip Ratio & 0.76 & 0.92 & 0.83 \\
(Female) & & & \\
FBS & 81 & 521 & $151.88 \pm 73.50$ \\
HbA1c & 6.1 & 14.6 & $8.01 \pm 2.08$ \\
ALT & 16 & 74 & $37.97 \pm 10.44$ \\
AST & 14 & 46 & $26.30 \pm 6.68$ \\
\hline
\end{tabular}

The Pearson correlation between FBS, HBA1c with serum aminotransferase levels were significantly positive. ALT correlated more significantly $(r=0.76$, $\mathrm{n}=5, \mathrm{p}<0.001)$. In this study, there were increase in serum aminotransferases levels with poor glycemic control, higher BMI and WHR. Results also showed that serum aminotransferases levels were higher among patients receiving insulin compared with 


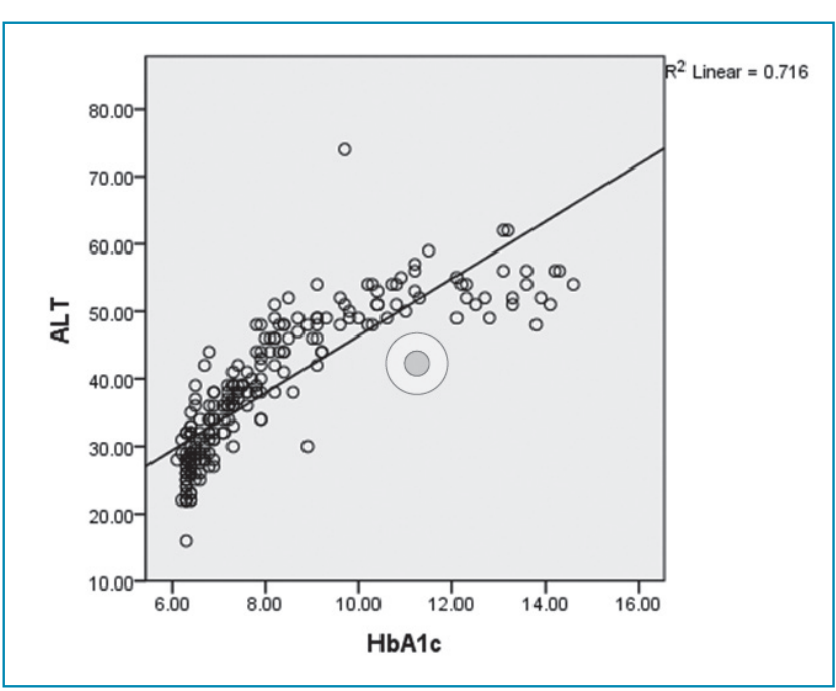

Fig. 1: Correlation of ALT with HbA1c patients receiving only Oral hypoglycemic agents. Most of the patients receiving only insulin had prior inadequate glucose control by OHA. Diabetic retinopathy was seen among six patients in older age group. All of them had nonproliferative diabetic retinopathy and had higher serum aminotransferases level.

\section{DISCUSSION}

Patients with type 2 diabetes mellitus have been reported to be associated with higher incidence of abnormal liver function test. Abnormal liver function test in diabetes can be attributed to several factors, the most common cause being NAFLD associated with insulin resistance and metabolic syndrome. Raised liver enzymes could be a marker of steatohepatitis in diabetic patients.

\section{Table 2: Comparative values of serum aminotransferases with BMI}

\begin{tabular}{llllll} 
Serum aminotransferases & BMI & N & Mean & Std. Deviation & P value \\
\multirow{2}{*}{ ALT } & $\geq 22.90$ & 136 & 42.9853 & 10.00295 & \\
& $<22.90$ & 100 & 31.1700 & 6.42919 & .001 \\
\multirow{2}{*}{ AST } & $\geq 22.90$ & 136 & 29.1985 & 6.73720 & \\
& $<22.90$ & 100 & 22.3800 & 4.14578 & .001 \\
\hline
\end{tabular}

Table 3: Comparative values of serum aminotransferases with WHR according to gender

\begin{tabular}{|c|c|c|c|c|c|c|}
\hline & Serum aminotransferases & WHR & $\mathbf{N}$ & Mean & Std. Deviation & P Value \\
\hline \multirow{4}{*}{ Male } & AST & $<0.89$ & 54 & 22.7407 & 4.59362 & \\
\hline & & $\geq 0.89$ & 40 & 31.7500 & 6.38809 & .001 \\
\hline & ALT & $<0.89$ & 54 & 31.6481 & 6.47246 & \\
\hline & & $\geq 0.89$ & 40 & 47.4000 & 8.21131 & .001 \\
\hline \multirow{4}{*}{ Female } & AST & $<0.82$ & 65 & 22.6615 & 4.04767 & \\
\hline & & $\geq 0.82$ & 77 & 29.0649 & 6.71671 & .001 \\
\hline & ALT & $<0.82$ & 65 & 31.6308 & 6.93174 & \\
\hline & & $\geq 0.82$ & 77 & 42.8831 & 9.95645 & .001 \\
\hline
\end{tabular}

Table 4: Comparative values of serum aminotransferases with Diabetic Retinopathy

\begin{tabular}{|c|c|c|c|c|c|}
\hline Serum aminotransferases & Fundoscopy & $\mathrm{N}$ & Mean & Std. Deviation & p value \\
\hline \multirow{2}{*}{ ALT } & $\begin{array}{c}\text { Non } \\
\text { Proliferative }\end{array}$ & 6 & 52.0000 & 4.04969 & \multirow{3}{*}{.001} \\
\hline & Normal & 230 & 37.6130 & 10.31228 & \\
\hline \multirow[t]{2}{*}{ AST } & $\begin{array}{c}\text { Non } \\
\text { Proliferative }\end{array}$ & 6 & 38.8333 & 5.67157 & \\
\hline & Normal & 230 & 25.9826 & 6.40071 & .001 \\
\hline
\end{tabular}

Table 5: Comparative values of serum aminotransferases among different treatment groups

\begin{tabular}{lcccccc} 
Serum aminotransferases & Treatment & N & Mean & Std. Deviation & P value \\
ALT & OHA & 204 & 35.7353 & 9.29423 & .001 \\
& Insulin & 32 & 52.2813 & 4.15998 & \\
AST & OHA & 204 & 25.0196 & 6.08799 & .001 \\
\hline
\end{tabular}


In our study, nearly two third of the patients were female. Most of the patients were in the age group of 41-50 years. It is also found that NAFLD is more prevalent in this age group. ${ }^{15}$ The mean serum aminotransferases level was within the reference range of our laboratory values. The mean FBS and HbA1c values were higher than normal which shows most patients in this study had poor glycemic control.

Idris et $a l,{ }^{16}$ compared liver function test in 50 type 2 diabetic patients and 30 normal control subjects in Sudan. The mean ALT, AST, gamma GT were reported to be significantly higher among diabetics compared to normal. In their study, the mean values were within the normal reference range. Similarly, in our study we found serum aminotransferase within our laboratory reference range however the subgroup analysis among different variables with serum aminotransferases while considering the normal cut off values for each variable showed significant differences among mean values. Both ALT and AST levels correlated significantly with increase in HbA1c and FBS. ALT correlated more significantly than AST $(r=0.76, n=5, p<0.001)$

The study done by Meybodi et al. ${ }^{17}$ found 1.6 times elevated ALT in type 2 diabetic patients than general population which was unrelated to age, BMI, glycemic control, triglyceride levels. Raised ALT was seen in patients with higher FBS level. Though there was positive correlation between ALT and FBS it was not statistically significant. In contrast to their finding our study showed statistically significant positive correlation between ALT and FBS.

Erby et $a l^{18}$ studied the prevalence of elevated ALT levels in the diabetic patients of US and found it in $7.8 \%$ of the diabetic. This was higher in obese (BMI>25) compared to that in non-obese $(10.6 \%$ vs $6.6 \%)$. Similar to their findings our study also showed higher serum aminotransferases level among patients with higher BMI.
The elevated ALT level was significantly related to BMI of $>25 \mathrm{~kg} / \mathrm{m}^{2}$ among the type 2 diabetic patients $(\mathrm{p}=0.01)$ in a study conducted in India. ${ }^{19}$ Positive correlation were also reported between glycemic control (FBS and PPBS) with the ALT level. Similar to their findings our study also showed positive correlation between FBS and ALT level.

Salmela et al $^{20}$ studied the liver function test of 175 patients without chronic liver diseases, where 57 $\%$ were found to have at least one abnormal LFT, $27 \%$ had at least two abnormal LFTs however the increase in liver function values were rarely more than two times of upper limit of normal. In their study they have found that the elevated ALT in diabetic patients was associated with increased BMI and poor glycemic control. Our study also showed similar association.

In our study serum aminotransferases level was assessed depending upon the treatment received by the diabetic patients. The mean aminotransferases level was significantly higher among patients who received only insulin compared to OHA. Most of the patients receiving only insulin had prior inadequate glucose control by OHA. So, the poor glycemic control in this group might be responsible for the elevated serum aminotransferases level.

Our study showed six patients with nonproliferative diabetic retinopathy had higher serum aminotransferases level which may be due to longer duration of diabetes with poor glycemic control. There are hardly any studies comparing serum aminotransferases level among the patients with diabetic retinopathy.

Thus, our study shows higher serum aminotransferases level in diabetic patients especially among the patients with poor glycemic controls. Our study also emphasizes on early detection of NAFLD in obese and diabetic patients by screening with serum aminotranferases.

\section{REFERENCES}

1. Amos AF, McCarty DJ, Zimmet P. The rising global burden of diabetes and its complications: estimates and projections to the year 2010. Diabet Med 1997; 14: 7-85.

2. Wild S, Roglic G, Green A, Sicree R, King H. Global prevalence of diabetes: estimates for the year 2000 and projections for 2030. Diabetes Care 2004; 27: 1047-53.

3. Subedi S, Subedi KU, Badhu BP. Doctor's role in early detection of diabetic retinopathy and prevention of blindness from its complications. $J$ Nepal Med Assoc 2005; 4: 26-30.

4. Singh DL, Bhattarai M. High prevalence of diabetes and impaired fasting glycaemia in urban Nepal. Diabet Med 2003; 20: 170-1.
5. Gupte P, Amarapurkar D, Agal S, Baijal R, Kulshrestha P, Pramanik S et al. Nonalcoholic steatohepatitis in type 2 diabetes mellitus. J Gastroenterol Hepatol 2004; 19: 854-8.

6. Choudhury J, Sanyal AJ. Insulin resistance and the pathogenesis of nonalcoholic fatty liver disease. Clin Liver Dis 2004; 8: 575-94.

7. Williams et al. Prevalence of NAFLD and Nash among a largely middle-aged population utilizing ultrasound and liver biopsy: A Prospective Study. Gastroenterology 2011; 140: 124-31

8. Vernon G, Baranova A, Younossi ZM. Systematic review: the epidemiology and natural history of non-alcoholic fatty liver disease and non-alcoholic steatohepatitis in adults. Aliment Pharmacol Ther 2011; 34: 274-85. 
9. Amarapurkar et al. How common is NAFLD in the Asia Pacific region and are there local differences? J Gastroenterol Hepatol 2007; 22: 788-93.

10. Hanley AJG, Williams K, Festa A et al. Elevations in Markers of Liver Injury and Risk of Type 2 Diabetes. Diabetes 2004; 53: 2623-32.

11. Chitturi S. NASH and insulin resistance: Insulin hypersecretion and specific association with the insulin resistance syndrome. Hepatology 2002; 35: 373-9.

12. Kelley DE, McKolanis TM, Hegazi RAF, Kuller LH, Kalhan SC. Fatty liver in type 2 diabetes mellitus: relation to regional adiposity, fatty acids, and insulin resistance. Am J Physiol Endocrinol Metab 2003; 285: 906-16.

13. Rensen SS, Slaats Y, Nijhuis J, Jans A, Bieghs V, Driessen A et al. Increased Hepatic Myeloperoxidase Activity in Obese Subjects with Nonalcoholic Steatohepatitis. Am J Pathol 2009; 175: 1473-82.

14. Marchesini G, Brizi M, Bianchi G, Tomassetti S, Bugianesi E, Lenzi M et al. Nonalcoholic Fatty Liver Disease: A Feature of the Metabolic Syndrome. Diabetes 2001; 50: 1844-50.
15. Caldwell SH, Oelsner DH, Iezzoni JC, Hespenheide EE, Battle EH, Driscoll CJ. Cryptogenic cirrhosis: clinical characterization and risk factors for underlying disease. Hepatology 1999; 29: 664-9.

16. Idris AS, Faisal K, Mekky H, Eldin B, Abdalla E, Ali KA. Liver function tests in type 2 Sudanese diabetic patients. Int'l J Nutri Metabolism 2011; 3: 17-21.

17. Meybodi MA, Afkhami-Ardekani M, Rashidi M. Prevalence of abnormal serum alanine aminotransferase levels in type 2 diabetic patients in Iran. Pakistan J Biol Sci 2008; 11: 2274-7.

18. Erbey JR, Silberman C, Lydick E. Prevalence of abnormal serum alanine aminotransferase levels in obese patients and patients with type 2 diabetes. Am J Med 2000; 109: 588-90.

19. Jayarama N, Sudha R. A study of non-alcoholic fatty liver disease (NAFLD) in type2 diabetes mellitus in a tertiary care centre, Southern India. J Clin Diagnostic Res 2012; 4: 11-17.

20. Salmela PI, Sotaniemi EA, Niemi M, Mäentausta O. Liver function tests in diabetic patients. Diabetes Care 1984; 7: 248-54. 